\title{
SOME NEW RESULTS ABOUT HAMMERSTEIN EQUATIONS ${ }^{1}$
}

\author{
BY HAIM BRÉZIS AND FELIX E. BROWDER
}

Communicated by S. S. Chern, August 1, 1973

Let $\Omega$ be a $\sigma$-finite measure space. Let $K$ be a (nonlinear) montone operator and let $(F u)(x)=f(x, u(x))$ be a Niemytski operator. We consider the Hammerstein type equation

$$
u+K F u=g .
$$

A detailed discussion and a complete bibliography about equation (1) can be found in [3]. The new feature about the results we present here is the fact that we do not assume any coercivity for $F$. When $F$ is monotone and $K$ maps $L^{1}(\Omega)$ into $L^{\infty}(\Omega)$, there is no growth restriction on $F$ either (cf. Theorem 1). The monotonicity of $F$ can be weakened when $K$ is compact (cf. Theorem 4). Also some of these results are valid for systems in the case where $F$ is the gradient of a convex function (cf. Theorem 5).

Assume

(2) $K$ is a monotone hemicontinuous mapping from $L^{1}(\Omega)$ into $L^{\infty}(\Omega)$ which maps bounded sets into bounded sets,

(3) $f(x, r): \Omega \times \boldsymbol{R} \rightarrow \boldsymbol{R}$ is continuous and nondecreasing in $r$ for a.e. $x \in \Omega$, and is integrable in $x$ for all $r \in \boldsymbol{R}$.

THEOREM 1. Under the assumptions (2) and (3), equation (1) has one cind only one solution $u \in L^{\infty}(\Omega)$ for every $g \in L^{\infty}(\Omega)$.

Uniqueness. Let $u_{1}$ and $u_{2}$ be two solutions of (1). By the monotonicity of $K$ we get

$$
\int_{\Omega}\left(u_{1}(x)-u_{2}(x)\right) \cdot\left(f\left(x, u_{1}(x)\right)-f\left(x_{1}, u_{2}(x)\right)\right) d x \leqq 0
$$

which implies that $f\left(x, u_{1}(x)\right)=f\left(x, u_{2}(x)\right)$ a.e. on $\Omega$ and therefore by (1), $u_{1}=u_{2}$.

In proving existence of $u$ we shall use the following

LEMMA 1. Let $X$ be a Banach space and let $K: X \rightarrow X^{*}$ and $F: X^{*} \rightarrow X$ be two monotone hemicontinuous operators. Let $\left\{u_{n}\right\} \subset X^{*},\left\{v_{n}\right\} \subset X$ and

AMS (MOS) subject classifications (1970). Primary 47H05, 47H15, 45G05.

1 Partially supported by NSF GP-28148. 
$\left\{w_{n}\right\} \subset X^{*}$ be three sequences such that

(4) $u_{n}$ converges to $u$ in $X^{*}$ for the weak* topology,

(5) $F\left(u_{n}\right)$ converges to $v$ in $X$ for the weak topology,

(6) $v_{n}$ converges to $v$ in $X$ for the weak topology,

(7) $K v_{n}$ converges to $g-u$ in $X^{*}$ for the weak* topology,

(8) $\left\langle w_{n}, F\left(u_{n}\right)\right\rangle-\left\langle K v_{n}, v_{n}\right\rangle \rightarrow 0$,

(9) $\left\langle g_{n}, F\left(u_{n}\right)\right\rangle \rightarrow\langle g, v\rangle$ where $g_{n}=u_{n}+w_{n}$.

Then $u+K F u=g$.

Proof of Lemma 1. We have

$$
\left\langle u_{n}-u, F\left(u_{n}\right)\right\rangle=\left\langle g_{n}-w_{n}-u, F\left(u_{n}\right)\right\rangle .
$$

By the monotonicity of $K$ we get

and thus

$$
\left\langle K v_{n}, v_{n}\right\rangle \geqq\left\langle K v_{n}, v\right\rangle+\left\langle K v, v_{n}-v\right\rangle
$$

By (8) we have

$$
\lim \inf \left\langle K v_{n}, v_{n}\right\rangle \geqq\langle g-u, v\rangle \text {. }
$$

$$
\lim \inf \left\langle w_{n}, F u_{n}\right\rangle \geqq\langle g-u, v\rangle .
$$

Consequently, $\lim \sup \left\langle u_{n}-u, F\left(u_{n}\right)\right\rangle \leqq 0$. Since $F$ is pseudomonotone (cf. [1]), we conclude that $v=F u$ and $\left\langle u_{n}, F\left(u_{n}\right)\right\rangle \rightarrow\langle u, v\rangle$. Also $\left\langle K v_{n}, v_{n}\right\rangle \rightarrow$ $\langle g-u, v\rangle$ since $\left\langle w_{n}, F\left(u_{n}\right)\right\rangle=\left\langle g_{n}-u_{n}, F\left(u_{n}\right)\right\rangle \rightarrow\langle g, v\rangle-\langle u, v\rangle$. Thus

$$
\lim \left\langle K v_{n}, v_{n}-v\right\rangle=0 \text {, }
$$

and again, since $K$ is pseudomonotone, we conclude that $g-u=K v=K F u$.

Proof of Theorem 1. By a shift we can always assume that $f(x, 0)=0$ and that $K 0=0$ (note that (1) can be written as $u+\tilde{K} \tilde{F} u=\tilde{g}$, where $\tilde{F} v=F v-F 0, \quad \tilde{K} v=K(v+F(0))-K F 0$ and $\tilde{g}=g-K F 0)$. Let $\Omega_{n}$ be an increasing sequence of finite measure subsets of $\Omega$ such that $\bigcup_{n} \Omega_{n}=\Omega$. Let $\chi_{n}$ be the characteristic function of $\Omega_{n}$. Let $F_{n}$ be $F$ truncated by $n$, i.e.,

$$
\begin{aligned}
f_{n}(x, r) & =f(x, r) & & \text { whenever }|f(x, r)|<n, \\
& =n f(x, r) /|f(x, r)| & & \text { whenever }|f(x, r)| \geqq n .
\end{aligned}
$$

The equation

$$
u_{n}+\chi_{n} K \chi_{n} F_{n}\left(u_{n}\right)=\chi_{n} g
$$

has a solution.

Indeed the mapping $K_{n}: v \mapsto \chi_{n} K \chi_{n} v$ is monotone hemicontinuous from $L^{2}(\Omega)$ into itself.

On the other hand, the (multivalued) operator $A$ defined on $L^{2}(\Omega)$ by

$$
A v=\left\{w \in L^{2}(\Omega) ; v(x)=\chi_{n}(x) f_{n}(x, w(x)) \text { a.e. on } \Omega\right\}
$$


is maximal monotone in $L^{2}(\Omega)$ and $D(A)$ is bounded in $L^{2}(\Omega)\left(|v|_{L^{2}} \leqq\right.$ $n\left(\text { meas } \Omega_{n}\right)^{1 / 2}, v \in D(A)$ ). Consequently, $R\left(A+K_{n}\right)=L^{2}(\Omega)$ (cf. [2]) and (10) has a solution.

Multiplying (10) through by $F_{n}\left(u_{n}\right)$ and using the monotonicity of $K$ we get

$$
\int_{\Omega} u_{n} \cdot F_{n}\left(u_{n}\right) d x \leqq \int_{\Omega} \chi_{n} g F_{n}\left(u_{n}\right) d x
$$

Let $C=2\|g\|_{L^{\infty}}$; we have

$$
\begin{aligned}
\int_{\Omega} u_{n} F_{n}\left(u_{n}\right) d x & =\int_{\left|u_{n}\right| \geqq C} u_{n} F_{n}\left(u_{n}\right) d x+\int_{\left|u_{n}\right|<C} u_{n} F_{n}\left(u_{n}\right) d x \\
& \geqq C \int_{\left|u_{n}\right| \geqq C}\left|F_{n}\left(u_{n}\right)\right| d x-C \int_{\left|u_{n}\right|<C}\left|F_{n}\left(u_{n}\right)\right| d x \\
& \geqq C \int_{\Omega}\left|F_{n}\left(u_{n}\right)\right| d x-2 C \int_{\left|u_{n}\right|<C}\left|F_{n}\left(u_{n}\right)\right| d x
\end{aligned}
$$

Using (11) we obtain

$$
\int_{\Omega}\left|F_{n}\left(u_{n}\right)\right| d x \leqq 4 \int_{\left|u_{n}\right| \leqq C}\left|F_{n}\left(u_{n}\right)\right| d x \leqq 4 \int_{\left|u_{n}\right| \leqq C}\left|f\left(x, u_{n}(x)\right)\right| d x \leqq C^{\prime}
$$

by assumption (3).

Going back to (10), we conclude that $\left\{u_{n}\right\}$ remains bounded in $L^{\infty}(\Omega)$. Therefore, by assumption (3), there is some function $h \in L^{1}(\Omega)$ such that

$$
\left|F_{n}\left(u_{n}\right)(x)\right| \leqq\left|f\left(x, u_{n}(x)\right)\right| \leqq h(x) \text { a.e. on } \Omega \text {. }
$$

We apply now Lemma 1 with $v_{n}=\chi_{n} F_{n}\left(u_{n}\right), w_{n}=\chi_{n} K v_{n}, g_{n}=\chi_{n} g$. By extracting a subsequence, we can always assume that

$u_{n}$ converges to $u$ weak ${ }^{*}$ in $L^{\infty}(\Omega)$,

$F\left(u_{n}\right)$ converges to $v$ weakly in $L^{1}(1)$,

$v_{n}$ converges to $v$ weakly in $L^{1}(\Omega)$,

$g_{n}$ converges to $g$ weak* in $L^{\infty}(\Omega)$.

\section{Hence}

$w_{n}$ converges to $g-u$ weak $*$ in $L^{\infty}(\Omega)$,

$K v_{n}$ converges to $g-u$ weak $^{*}$ in $L^{\infty}(\Omega)$.

It remains to verify (8) and (9). We have

$$
\begin{aligned}
\left\langle w_{n}, F\left(u_{n}\right)\right\rangle & =\int_{\Omega} \chi_{n} K v_{n} \cdot F\left(u_{n}\right) d x=\int_{\Omega} K v_{n} \chi_{n} F\left(u_{n}\right) d x \\
& =\int_{\Omega} K v_{n} \cdot v_{n} d x+\int_{\Omega} \chi_{n} K v_{n}\left(F\left(u_{n}\right)-F_{n}\left(u_{n}\right)\right) d x
\end{aligned}
$$


The last term can be bounded by

$$
C \int_{\left|F\left(u_{n}\right)\right|>n}\left|F u_{n}\right| d x \leqq C \int_{|h|>n}|h(x)| d x
$$

which tends to zero as $n \rightarrow+\infty$ and (8) follows.

Finally (9) holds since

$$
\left\langle g_{n}, F\left(u_{n}\right)\right\rangle=\int_{\Omega} \chi_{n} g F\left(u_{n}\right) d x=\int_{\Omega} g F\left(u_{n}\right) d x+\int_{\Omega}\left(\chi_{n}-1\right) g F\left(u_{n}\right) d x,
$$

and the last term goes to zero by Lebesgue's theorem.

THEOREM 2 (CONTINUOUS DEPENDENCE). Under the assumptions (2) and (3), $F(I+K F)^{-1}$ is strongly continuous from $L^{\infty}(\Omega)$ into $L^{1}(\Omega)$ and $(I+K F)^{-1}$ is demicontinuous (from $L^{\infty}(\Omega)$ strong into $L^{\infty}(\Omega)$ weak ${ }^{*}$ ). If in addition $K$ is strongly continuous from $L^{1}(\Omega)$ into $L^{\infty}(\Omega)$, then $(I+K F)^{-1}$ is strongly continuous from $L^{\infty}(\Omega)$ into $L^{\infty}(\Omega)$.

Proof. We shall prove a slightly stronger result. Let $g_{n}$ be a bounded sequence in $L^{\infty}(\Omega)$ such that $g_{n} \rightarrow g$ a.e. on $\Omega$. Let $u_{n}=(I+K F)^{-1} g_{n}$ and let $u=(I+K F)^{-1} g$. We are going to show that $F\left(u_{n}\right) \rightarrow F(u)$ in $L^{1}(\Omega)$.

We know, from the proof of Theorem 1 , that $\left\{u_{n}\right\}$ is bounded in $L^{\infty}(\Omega)$ and there is some $h \in L^{1}(\Omega)$ such that $\left|F\left(u_{n}\right)\right| \leqq h$ a.e. on $\Omega$. Since

$$
\int_{\Omega}\left(u_{n}-u\right)\left(F\left(u_{n}\right)-F(u)\right) d x \leqq \int_{\Omega}\left(g_{n}-g\right)\left(F\left(u_{n}\right)-F(u)\right) d x
$$

and the right hand side goes to zero by Lebesgue's theorem, we can extract a subsequence such that

$$
\left(u_{n_{k}}-u\right)\left(F\left(u_{n_{k}}\right)-F(u)\right) \rightarrow 0 \text { a.e. on } \Omega \text {. }
$$

Consequently, $F\left(u_{n_{k}}\right) \rightarrow F(u)$ a.e. on $\Omega$ and hence $F\left(u_{n_{k}}\right) \rightarrow F(u)$ in $L^{1}(\Omega)$. By the uniqueness of the limit we conclude that $F\left(u_{n}\right) \rightarrow F(u)$ in $L^{1}(\Omega)$.

Using similar arguments, we can prove some variants of Theorem 1.

THEOREM 3. Assume $K$ is monotone, hemicontinuious and bounded from $L^{p^{\prime}}(\Omega)$ into $L^{p}(\Omega)$. Assume $f(x, r): \Omega \times \boldsymbol{R} \rightarrow \boldsymbol{R}$ is continuous and nonincreasing in $r$ for a.e. $x \in \Omega$ and is measurable in $x$ for all $x \in \boldsymbol{R}$, and satisfies

$$
|f(x, r)| \leqq c(x)+c_{0}|r|^{p-1} \quad \text { a.e. } x \in \Omega, \text { for all } r \in \boldsymbol{R}
$$

where $c \in L^{p^{\prime}}(\Omega)$.

Then (1) has a unique solution $u \in L^{p}(\Omega)$ for every $g \in L^{p}(\Omega)$.

THEOREM 4. Assume $K$ is monotone, hemicontinuous from $L^{1}(\Omega)$ into $L^{\infty}(\Omega)$ and maps bounded sets of $L^{1}(\Omega)$ into compact sets of $L^{\infty}(\Omega)$. 
Assume $f(x, r)$ is continuous in $r$ for a.e. $x \in \Omega$ and there exists $M$ such that

$$
(f(x, r)-f(x, 0)) r \geqq 0 \text { for a.e. } x \in \Omega \text { and for all }|r| \geqq M \text {. }
$$

Suppose $f(x, r)$ is measurable in $x$ for all $r \in \boldsymbol{R}$ and for every constant $C$,

$$
\int_{|r| \leqq C}|f(x, r)| \text { is integrable. }
$$

Then (1) has a solution $u \in L^{\infty}(\Omega)$ for every $g \in L^{\infty}(\Omega)$.

The case of systems. Assume

(13) $K$ is monotone hemicontinuous and bounded from $L^{1}\left(\Omega ; R^{n}\right)$ into $L^{\infty}\left(\Omega ; \boldsymbol{R}^{n}\right)$.

(14) $f(x, r): \Omega \times \boldsymbol{R}^{n} \rightarrow \boldsymbol{R}^{n}$ is continuous in $\boldsymbol{r}$ for a.e. $x \in \Omega$ and trimonotone in $r$, i.e., for a.e. $x \in \Omega$ and for any sequence $r_{0}, r_{1}, r_{2}, r_{3}=r_{0}$ we have

$$
\sum_{i=1}^{3}\left(f\left(x, r_{i}\right), r_{i}-r_{i-1}\right) \geqq 0
$$

(for example, the gradient of a convex function is trimonotone, see [4]).

(15) $f(x, r)$ is measurable in $x$ for all $r \in \boldsymbol{R}$ and for every constant $C$

$$
\int_{|r| \leqq C}|f(x, r)| \text { is integrable. }
$$

THEOREM 5. Under the assumptions (13), (14), (15), equation (1) has a unique solution $u \in L^{\infty}\left(\Omega ; \boldsymbol{R}^{n}\right)$ for every $g \in L^{\infty}\left(\Omega ; \boldsymbol{R}^{n}\right)$.

In order to bound $F u$ in $L^{1}$, we use the following

Lemma 2. Assume (14) and (15) hold. Then for any constant $\rho>0$, there exists $h_{\rho} \in L^{1}(\Omega)$ such that

$$
\rho|f(x, r)| \leqq(f(x, r)-f(x, 0), r)+h_{\rho}(x) \text { for a.e. } x \in \Omega \text {, all } r \in \boldsymbol{R}^{n} .
$$

Uniqueness follows from the following

LEMMA 3. Assume $B$ is continuous and trimonotone from a Hilbert space $H$ into itself. Let $u, v \in H$ be such that

Then $B u=B v$.

$$
(B u-B v, u-v)=0 .
$$

Along the same lines one can prove the following lemma which leads to stability results.

LEMMA 4. Assume $B$ is trimonotone and Hölder continuous with exponent $\alpha \leqq 1$ (i.e., $|B u-B v| \leqq L|u-v|^{\alpha}$ for all $u, v \in H$ ). 
Then there exists a constant $k>0$ such that

$$
(B u-B v, u-v) \geqq k|B u-B v|^{1+1 / \alpha} \text { for all } u, v \in H .
$$

\section{REFERENCES}

1. H. Brézis, Équations et inéquations non linéaires dans les espaces vectoriels en dualité, Ann. Inst. Fourier (Grenoble) 18 (1968), fasc. 1, 115-175. MR 42 \#5113.

2. F. E. Browder, Nonlinear maximal monotone operators in Banach spaces, Math. Ann. 175 (1968), 89-113. MR 36 \#6989.

3. - Nonlinear functional analysis and nonlinear integral equations of Hammerstein and Urysohn type, Contributions to Nonlinear Functional Analysis, E. Zarantonello ed. Academic Press, New York.

4. R. T. Rockafellar, Characterization of subdifferentials of convex functions, Pacific J. Math. 17 (1966), 497-510. MR 33 \#1769.

Department of Mathematics, University of Chicago, Chicago, Illinois 60637 